\title{
Could Resilience Predict the Outcome of Psychiatric Rehabilitation Patients?
}

\author{
A Pilot Study* \\ Birk Engmann ${ }^{\#}$ \\ Department of Neurology and Psychosomatics, Brandis Rehabilitation Centre, Am Wald, Brandis, Germany \\ Email: "oa.engmann@fachklinikum-brandis.de
}

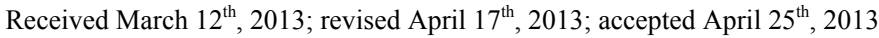

Copyright (C) 2013 Birk Engmann. This is an open access article distributed under the Creative Commons Attribution License, which permits unrestricted use, distribution, and reproduction in any medium, provided the original work is properly cited.

\begin{abstract}
Background: Individual resistance to burdens such as stress, adversity, and even disasters is called "resilience". Whereas most studies of resilience investigate post-traumatic stress disorder, fewer studies treat anxiety and depressiveness in mentally healthy populations. The present pilot study investigates whether resilience lessens the severity of depressive symptoms. Aim was to figure out whether further research is needed on the relation of resilience both to depression and to rehabilitation from it in regard to fitness for work. Design/Setting/Population: A retrospective, non-blind, non-randomized analysis of charts of 503 stationary inpatient-patients was performed. Patients without age limits who suffered from diseases of the depressive spectrum (F32, F33) and also adjustment disorder (F43.2), dysthymia (F34.1), and neurasthenia (F48) according to ICD 10 were included. BDI and resilience scale, state of fitness or unfitness for work, were analysed with correlation analysis and descriptive statistics by the SPSS program. Results: Analysis revealed that resilience and depressive symptoms are inversely related. The higher the resilience the lower the BDI score, indicating a lower burden of depressive symptoms. Resilience also seems to influence important outcome factors of rehabilitation such as reintegration in work and remission of depressive symptoms. Patients who either were fit for work at the beginning of rehabilitation or were dismissed as fit for work had higher resilience scores than those who were unfit for work. Conclusion: This pilot study encourages further investigation of the relation of resilience not only to depressive disorders, but also to rehabilitation and social reintegration after depression.
\end{abstract}

Keywords: Resilience; Rehabilitation; Depression; BDI

\section{Introduction}

Psychic diseases are being diagnosed more often, and becoming more prominent in absenteeism from work. Therefore, investigating factors which affect rehabilitation from psychic disease may help to assess outcome. Resilience could be one of such factors. According to Rutter (2006), "resilience is an interactive concept that refers to a relative resistance to environmental risk experiences, or the overcoming of stress or adversity. As such, it differs from both social competence and positive mental health. Resilience differs from traditional concepts of risk and protection in its focus on individual variations in response to comparable experiences". Others such as Luthar et al. (2006) and Cicchetti (2010) emphasize an additional influence of neurobiological and genetic factors.

Most of the studies focused on the relationship of posttraumatic stress disorder and resilience, such as Mealer et al. (2012) who showed that nurses with higher resilience could better cope with circumstances at intensive care units. Also, coping with disasters such as bombings was investigated. According to a study of disaster survivors in North and Cloninger (2012), de-

\footnotetext{
*Conflict of interest: The author declares that there is no conflict of interest. \#Corresponding author.
}

pression was not a robust marker of low resilience in a study of disaster survivors. Contrary to that, occurrence of posttraumatic stress disorder was associated with low resilience. A similar finding is in Ahmand et al. (2010), which points out that earthquake survivors with higher resilience scores had less posttraumatic symptom levels. Peres et al. (2011) investigated police officers with traumatic memories by fMRI scan. Policemen with high resilience, or those who were in a psychotherapy group, had decreased amygdala activity during traumatic memory retrieval in comparison to those without current psychotherapy.

Fewer studies dealt with the question of how depressive disorders relate to resilience. Hjemdal et al. (2011) compared resilience with anxiety and depression scales in psychically healthy teenagers in highschools in Norway. The authors showed that higher resilience is connected with lower scores for depression, anxiety, stress and compulsory behaviour. Pierini and Stuifbergen (2010) investigated resilience factors and depressiveness in patients with post-polio syndrome. They found spiritual growth as the main predictor of lesser depressive symptoms. A Korean study compared a healthy population with psychiatric outpatients. "Greater resilience was found to be associated with less perceived stress, anxiety and depression 
and with higher levels of positive affect and purpose in life", according to the authors (Jung et al., 2012).

In somatic diseases, resilience also improves outcome as shown in a study by Strauss et al. (2007) of cancer patients with fatigue who received radiation therapy. Furthermore, elderly people with higher resilience have fewer subjective body complaints, as reported in a study by Leppert et al. (2005).

All in all, previous studies with regard both to psychic and somatic symptoms showed the beneficial influence of resilience for a lower burden of psychic symptoms.

The present study investigates following hypotheses:

1) Patients with high resilience have lower BDI score at beginning of rehabilitation.

2) Patients with high resilience have more remissions of depressive symptoms within 3 weeks of starting rehabilitation than patients with low resilience.

3) Patients with high resilience are more likely to be classified as "fit for work" after rehabilitation.

\section{Methods}

This is a pilot study to assess further needs and possibilities for investigation. A retrospective, non-blind, non-randomized analysis of charts of stationary patients, i.e. only inpatient, no outpatient or daycare rehabilitation, from August 2011 to December 2011, was performed. In total, 503 cases fulfilled criteria for inclusion and exclusion (see below). The duration of rehabilitation lasted from 3 weeks to 5 weeks. All patients received multimodal treatment of both psychotherapy in oneto-one and group conversations, and furthermore, physiotherapy.

Included in the analysis were all diseases of the depressive spectrum (F32, F33) and also adjustment disorder (F43.2), dysthymia (F34.1), and neurasthenia (F48) according to ICD 10. Excluded were depressive states in bipolar disorders (F30, F31), schizophrenia (F20-F29), alcohol and drug addiction (F10-F19), brain diseases such as dementia or Parkinson's.

In Brandis hospital, BDI and resilience scale are standard tests for all patients. Remission or response in BDI is used as an outcome marker of a successful rehabilitation.

The following assessment criteria of Beck Depression Inventory (BDI) were used:

$\mathrm{BDI} \leq 10$ : no depressive symptoms.

BDI $=11-17$ : mild to moderate depressive symptoms.

BDI $\geq 18$ : clinically relevant depressive symptoms (Hautzinger et al., 1995).

Remission is defined as $\mathrm{BDI} \leq 10$. Response is defined as BDI score reduction of $50 \%$.

BDI's were filled out at beginning of rehabilitation and after 3 weeks.

For the assessment of resilience, the 11-item resilience scale RS-11 was used. This is a short version of 25-item resilience scale of Wagnild and Young (1993). It was modified and translated into German by Schumacher et al. (2005). Minimal score which stands for lowest resilience is 11 (1 point for each item), highest score is 77 ( 7 points for each item) which stands for a very high resilience. Patients filled out this resilience scale at beginning of rehabilitation.

Statistical analysis was conducted with SPSS.

\section{Results}

$34.4 \%(n=173)$ of the patients were male, $65.6 \%(n=330)$ were female (total: $\mathrm{n}=503$ ).

Minimum age was 19 years, maximum age 84 years. Mean age 51.8 years, median age 52.0 years (SD: 11.2). During rehabilitation psychiatric medication was altered in $21.8 \%(\mathrm{n}=110)$ of the subjects. Medication was stable in $77.8 \%(\mathrm{n}=393)$. No data in $0.4 \%(\mathrm{n}=2)$.

Is there a correlation between resilience and BDI score at beginning of rehabilitation? In 448 of 503 cases all data were available. Pearson correlation coefficient is -0.628 ( $p<$ 0.0001).

The graph (Figure 1) shows a good reverse correlation between resilience score and BDI at beginning of rehabilitation.

Another question is "does resilience influence duration of absenteeism from work by sick note?"

At the beginning of rehabilitation (Figure 2), 46.5\% $(n=235)$ of the patients were absent from work by sick note, and only $32.3 \%(\mathrm{n}=163)$ were fit for work. Others were old age and disability pensioners $(20.8 \%, \mathrm{n}=105)$.

At dismissal from rehabilitation $48.9 \%(n=247)$ were still counted as being unfit for work, and $29.9 \%$ were dismissed as fit for work (pensioners $20.8 \%, \mathrm{n}=105$ ).

Before rehabilitation, the longest period of being unfit for work by a sick note was 1540 days, the minimal was 2 days, and the mean was 212.5 days, and the median was 180.0 days (SD: 161.7). That means that the average amount of time that a person was unfit for work by a sick note before rehabilitation was approximately 4 months!

Is there a relation between fit or unfit for work and resilience?

First, resilience scores were tested for normal distribution by Kolmogorov-Smirnov-Test (2-tailed $p=0.370$; near by alpha $=$ $0.4)$ which was fulfilled.

The mean resilience score in the whole sample (448 patients, 57 missing) is 46.8 (median 47, SD: 15.5).

Patients who came to rehabilitation unfit for work had a mean resilience score of 43.1 (median 43.5, SD: 14.8; $n=214$, missing resilience score in 21 subjects). Contrary to that, patients who came fit for work to rehabilitation had a mean resilience score of 51.1 (median 52.0, $\mathrm{n}=145$, SD: 14.6; missing resilience score in 18 subjects).

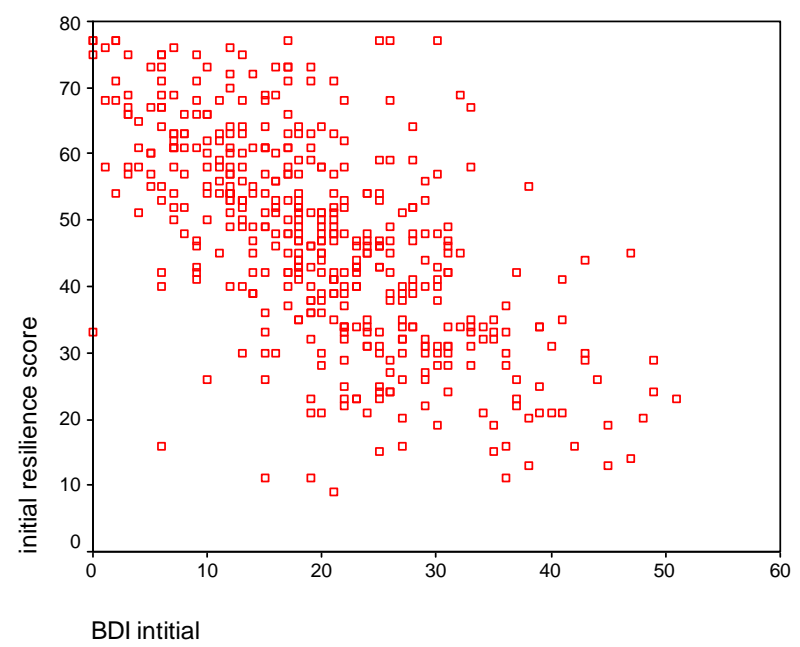

Figure 1.

Correlation between BDI and resilience score at beginning of rehabilitation. 


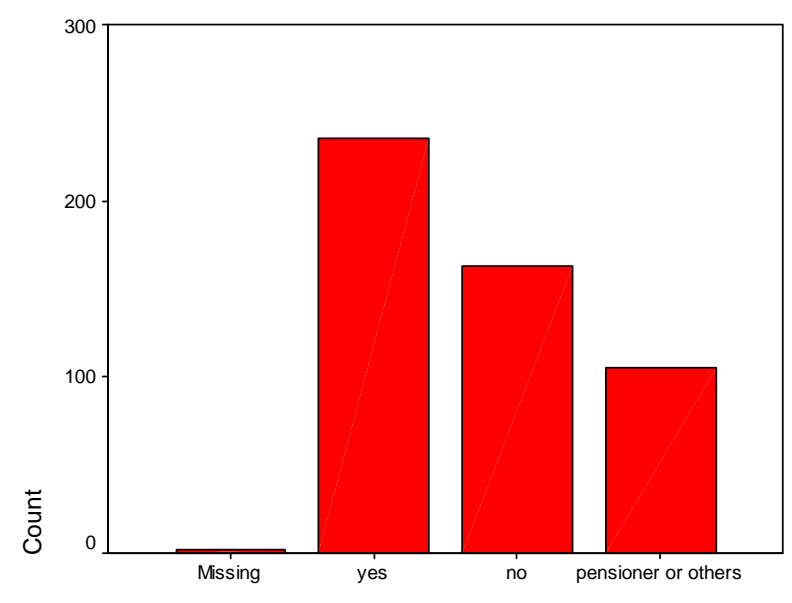

unfit for work at admission

Figure 2.

Status of being fit or unfit for work at the beginning of rehabilitation.

Similar findings exist for dismissal. In patients who were released unfit for work from rehabilitation mean resilience score was 42.97 (median 43.0, $\mathrm{n}=223$, SD: 14.8; missing resilience score in 14 subjects). Patients who were released fit for work had a mean resilience score of 51.99 (median 52.50, $\mathrm{n}=136$, SD: 14.1 ; missing resilience score in 15 subjects).

In conclusion, there is a trend that patients who either came fit for work or left rehabilitation fit for work had higher resilience scores.

Does resilience predict outcome of depressive symptoms?

Patients who had a remission after 3 weeks rehabilitation had a mean resilience score of 53.56 (median 54.0, SD: 12.3; $\mathrm{n}=61$, 0 missing).

Patients who had a response but no remission after 3 weeks rehabilitation had a mean resilience score of 40.11 (median 39.0, $\mathrm{n}=17$, SD: 7.7; 0 missing).

Patients who had neither remission nor response ("no effort") had a mean resilience score of 41.77 (median 42.0, $\mathrm{n}=252$, SD: 14.4; 4 missing).

In conclusion, a trend could be described that patients with remission are accompanied by higher resilience compared to patients with response or "no effort".

\section{Discussion}

The mean resilience score (46.8) in the whole sample is lower than the medium resilience score of a healthy population (58.03 according to Schumacher et al., 2005).

The study revealed a good reverse correlation between resilience and BDI. The higher the resilience the lower the BDI score, indicating a lower burden of depressive symptoms.

Two outcome items were investigated in relation to resilience: being fit or unfit for work and remission of depressive symptoms. There is a trend that patients who either were fit for work at the beginning of rehabilitation or were dismissed as fit for work had higher resilience scores that those who were unfit for work. The same is for patients with higher resilience who were more likely to have a remission than those with lower resilience. This finding could only be described as a trend because number of subjects in subgroups differs to a high degree and thus could lead to bias.

Patients with depressive disorders in rehabilitation have al- ready been absent from work approximately 4 months before the beginning of rehabilitation. This raises a question of when symptoms may be considered chronic.

It is interesting that there is no major effect of rehabilitation in relation to whether patients are fit or unfit for work before and after rehabilitation and the outcome is counterintuitive, in that there was a slight increase of patients judged unfit for work after rehabilitation. Perhaps this outcome may be explained by differences between the physicians responsible for admitting the patient and the physicians of the rehabilitation clinic, in that they may have different criteria for being fit for work. Another question is if different approaches of involved physicians affect ICD-10 criteria and thus inclusion criteria. Other limitation factors could be the relatively small sample size, or the severity of the disease which goes with prolonged absenteeism from work before rehabilitation. Furthermore the study has no follow-up to assess the duration of absenteeism after dismissal nor does it contain information about how many patients had been included in a gradual reintegration program (called "stufenweise Wiedereingliederung" or "Hamburg model") in the old working place. This program deems one unfit for work until one achieves a full number of working hours. Such a program avoids the oversimplification of defining the value of rehabilitation entirely by results present at the time of dismissal.

Another factor which weakens the results of the present study is that psychiatric medications were altered during rehabilitation. In addition to it, even cases with no alteration during rehabilitation could benefit from delayed effects of a medication which had been started before rehabilitation. Moreover, patients did not receive a completely "superposable" psychological and physical treatment. Also, different age groups could lead to a bias in the study.

In conclusion, the present pilot study encourages further investigation of the relation of resilience to depressive disorders, and rehabilitation from them, and reintegration in social life.

\section{Acknowledgements}

Professor Dr. Wilfried Mau, Institute of Rehabilitation Medicine, University of Halle, Germany, and Professor Dr. Gerhard Schmid-Ott, Berolina Clinic, Bad Oeynhausen, Germany, for introduction to the topic of resilience.

\section{REFERENCES}

Ahmand, S., Feder, A., Lee, E. J., Wang, Y., Southwick, S. M., Schlackman, E., Buchholz, K., Alonso, A., \& Charney, D. S. (2010). Earthquake impact in a remote South Asian population: Psychosocial factors and posttraumatic symptoms. Journal of Traumatic Stress, 23, 408-412.

Cicchetti, D. (2010). Resilience under conditions of extreme stress: A multilevel perspective. World Psychiatry, 9, 145-154.

Hautzinger, M., Bailer, M., Worall, H., \& Keller, F. (1995). BeckDepressionis-Inventar (BDI). Testhandbuch (2nd ed.). Bern: Hans Huber.

Hjemdal, O., Vogel, P. A., Solem, S., Hagen, K., \& Stiles, T. C. (2011). The relationship between resilience and levels of anxiety, depression, and obsessive-compulsive symptoms in adolescents. Clinical Psychology \& Psychotherapy, 18, 314-321. doi:10.1002/cpp.719

Jung, Y. E., Min, J. A., Shin, A. Y., Han, S. Y., Lee, K. U., Kim, T. S., Park, J. E., Choi, S. W., Lee, S. H., Choi, K. S., Park, Y. M., Woo, J. M., Bhang, S. Y., Kang, E. H., Kim, W., Yu, J. J., \& Chae, J. H. (2012). Positiveness research team of Korea: The Korean version of the connor-davidson resilience scale: An extended validation. Stress 


\section{B. ENGMANN}

Health, 28, 319-326. doi:10.1002/smi.1436

Leppert, K., Gunzelmann, T., Schumacher, J., Strauss, B., \& Brähler, E. (2005). Resilience as a protective personality characteristic in the elderly. Psychotherapie, Psychosomatik, Medizinischle Psychologier, 55, 365-369. doi:10.1055/s-2005-866873

Luthar, S. S., Sawyer, J. A., \& Brown, P. J. (2006). Conceptual issues in studies of resilience: Past, present, and future research. Annals of the New York Academy of Sciences, 1094, 105-115. doi:10.1196/annals.1376.009

Mealer, M., Jones, J., \& Moss, M. (2012). A qualitative study of resilience and posttraumatic stress disorder in United States ICU nurses. Intensive Care Medicine, 38, 1445-1451. doi:10.1007/s00134-012-2600-6

North, C. S., \& Cloninger, C. R. (2012) Personality and major depresssion among directly exposed survivors of the Oklahoma city bombing. Depression Research and Treatment, 2012, Article ID: 204741.

Peres, J. F., Foerster, B., Santana, L. G., Fereira, M. D., Nasello, A. G., Savoia, M., Moreira-Almeida, A., \& Lederman, H. (2011). Police officers under attack: Resilience implications of an fMRI study. Journal of Psychiatric Research, 45, 727-734. doi:10.1016/j.jpsychires.2010.11.004

Pierini, D., \& Stuifbergen, A. K. (2010). Psychological resilience and depressive symptoms in older adults diagnosed with post-polio syndrome. Rehabilitation Nursing, 35, 167-175.

doi:10.1002/j.2048-7940.2010.tb00043.x

Rutter, M. (2006). Implications of resilience concepts for scientific understanding. Annals of the New York Academy of Sciences, 1094, 1-12. doi:10.1196/annals.1376.002

Schumacher, J., Leppert, K., Gunzelmann, Th., Strauß, B., \& Brähler, E. (2005). Die resilienzskala-Ein fragebogen zur erfassung der psychischen widerstandsfähigkeit als personmerkmal. Zeitschrift für klinische Psychologie, Psychiatrie und Psychotherapie, 53, 16-19.

Strauss, B., Brix, C., Fischer, S., Leppert, K., Füller, J., Roehrig, B., Schleussner, C., \& Wendt, T. G. (2007). The influence of resilience on fatigue in cancer patients undergoing radiation therapy (RT). Journal of Cancer Research and Clinical Oncology, 133, 511-518. doi:10.1007/s00432-007-0195-Z

Wagnild, G. M., \& Young, H. M. (1993). Development and psychometric evaluation of the Resilience Scale. Journal of Nursing Measurement, 1, 165-178. 